\title{
BAP1 Germline Mutations in Finnish Patients with Uveal Melanoma
}

\section{Turunen, Joni A.}

2016-05

Turunen , J A , Markkinen , S , Wilska , R , Saarinen , S, Raivio , V , Tall , M , Lehesjoki , A-E \& Kivela , T T 2016, ' BAP1 Germline Mutations in Finnish Patients with Uveal Melanoma ', Ophthalmology , vol. 123 , no. 5 , pp. 1112-1117 . https://doi.org/10.1016/j.ophtha.2016.01.008

http://hdl.handle.net/10138/224018

https://doi.org/10.1016/j.ophtha.2016.01.008

publishedVersion

Downloaded from Helda, University of Helsinki institutional repository.

This is an electronic reprint of the original article.

This reprint may differ from the original in pagination and typographic detail.

Please cite the original version. 


\section{BAP1 Germline Mutations in Finnish Patients with Uveal Melanoma}

Joni A. Turunen, MD, PhD, ${ }^{1,2} *$ Salla Markkinen, $\mathrm{MSc},{ }^{2}, *$ Rosi Wilska, MD ${ }^{1}$ Silva Saarinen, MD, PhD, ${ }^{3}$ Virpi Raivio, MD, PhD, ${ }^{1}$ Martin Täll, MD, ${ }^{1}$ Anna-Elina Lehesjoki, MD, PhD, ${ }^{2,4}$ Tero T. Kivelä, MD, PhD ${ }^{1}$

Purpose: Germline mutations of the BRCA1-associated protein-1 gene (BAP1) predispose carriers to uveal melanoma. We report the population-based frequency of germline pathogenic variants of BAP1 in Finnish patients with uveal melanoma who live in a high-risk region for this cancer.

Design: Cohort study.

Participants: In Finland, uveal melanomas are treated centrally in the Ocular Oncology Service, Helsinki University Hospital. We collected clinical data and genomic DNA from 148 of 188 consecutive patients diagnosed from January 2010 through December 2012. Seven of these patients from 6 families had a history of uveal melanoma in 1 relative, and 2 patients from 2 additional families had such a history in 2 relatives.

Methods: Sequencing BAP1.

Main Outcome Measures: Pathogenic variants in BAP1.

Results: We found 2 different pathogenic variants in $B A P 1$ in 3 patients. Two patients had a single nucleotide insertion in exon 14 resulting in a shift of reading frame. Both had a family history of uveal melanoma in at least 1 relative. One patient without a family history of uveal melanoma had a single nucleotide substitution in the conserved splice donor site of intron 2. BAP1 cancer predisposition syndrome-related cancers were present in all 3 families. The overall frequency of BAP1 pathogenic variants was $2.0 \%(3 / 148 ; 95 \%$ confidence interval, $0.4-5.8)$, the frequency among patients 50 years of age or younger was $3.6 \%(1 / 28 ; 95 \%$ confidence interval, $0.1-18$ ), and a pathogenic variant was detected in 2 of 8 families with a history of uveal melanoma.

Conclusions: The frequency of BAP1 germline pathogenic variants in consecutive Finnish patients with uveal melanoma who come from a high-risk region for the development of this cancer is comparable with reports from other populations. Ophthalmology 2016;123:1112-1117 ( 2016 by the American Academy of Ophthalmology.

Uveal melanoma is, after retinoblastoma, the second most common primary intraocular cancer in the world. ${ }^{1}$ It metastasizes in approximately one half of patients. ${ }^{2}$ Uveal melanoma develops from melanocytes in the choroid, ciliary body, or iris. The cause of this tumor is not well understood. For unknown reasons, the incidence of uveal melanoma is high in northern latitudes. ${ }^{3}$ In Finland, approximately 65 new uveal melanomas are diagnosed annually, yielding a crude incidence of 12 per 1 million. The age-standardized incidence in Nordic countries ranges from 6.1 to 8.6 per 1 million. ${ }^{4}$ Uveal melanoma is usually sporadic, but cases of familial uveal melanoma comprise $0.6 \%$ to $6 \%$ of reported series with this cancer.,

It is well known that loss of 1 chromosome 3 (monosomy 3 ) in tumor tissue is strongly associated with development of aggressive uveal melanoma. ${ }^{7,8}$ The breast cancer 1 (BRCA1)-associated protein-1 (BAPl) gene is located in chromosome 3p21.1. Both germline ${ }^{9,10}$ and somatic ${ }^{9}$ mutations of BAPl have been implicated in tumor progression. $B A P 1$ is mutated in most metastasizing uveal melanomas. ' Germline mutations of BAPl predispose carriers to uveal melanoma, cutaneous melanoma, melanocytic BAPl-mutated atypical intradermal tumors, mesothelioma, renal cell cancer, and possibly some other tumor types..$^{9,11,12}$ The BAPl mutation-associated cancer risk is inherited in an autosomal dominant pattern with incomplete penetrance for any given cancer type. ${ }^{12}$

The BAP1 pathogenic variant frequency among patients with uveal melanoma has been estimated to be $2 \%$ to $3 \%$ in unselected populations. ${ }^{13}$ A recent referral-based study with 507 patients from the United States reported a mutation frequency of $1.6 \% .{ }^{14}$ In a population-based Australian study of 66 patients diagnosed with uveal melanoma at the age of 50 years or younger or with bilateral uveal melanoma at any age, mutation frequency was $3.0 \% .^{15}$ Herein we report the frequency of $B A P 1$ germline pathogenic variants in a consecutive, population-based cohort of patients with uveal melanoma diagnosed during a 3 -year period in Finland, a high-risk region for the development of this cancer.

\section{Methods}

\section{Patients}

In Finland, all uveal melanoma patients are referred to the Ocular Oncology Service, Helsinki University Hospital. Eligible for this study were all consecutive Finnish patients diagnosed with uveal 
melanoma from January 1, 2010, through December 31, 2012. Patients were invited to participate beginning on November 5, 2012.

During the study period, 193 new patients were diagnosed with primary uveal melanoma. Five patients were excluded because they were not originally from Finland. In addition, 13 patients did not respond to the invitation, 4 patients elected not to participate in the study, and 23 patients had died before they could be enrolled, 18 of them of metastatic uveal melanoma and 1 patient of a cytologically confirmed oat-cell lung carcinoma.

We collected clinical data and peripheral blood samples from the 148 consecutive patients who consented to participate in the study. This included 7 patients with a history of uveal melanoma in 1 relative and 2 patients with 2 such relatives, representing 8 different families. The inclusion rate was $79 \%$ of all 188 eligible patients and the participation rate was $90 \%$ of the 165 patients who could be invited to participate. The clinical characteristics of the patients at the time of diagnosis are presented in Table 1 . The project was approved by the appropriate institutional review board and followed the tenets of the Declaration of Helsinki. Written informed consent was obtained from all participants.

\section{Sequencing and Sequence Analysis}

Genomic DNA was extracted from peripheral blood using standard methods. All 17 coding exons and exon-intron junctions of $B A P 1$ (reference sequence, ENSG00000163930; available at: www.ensembl.org) were sequenced from polymerase chain reactionamplified fragments. The polymerase chain reaction conditions and primer sequences are available from the authors upon request. The BigDye Terminator version 3.1 Cycle Sequencing Kit (Applied Biosystems, Foster City, CA) and an ABI 3730 DNA Analyzer (Applied Biosystems) were used to sequence the purified polymerase chain reaction products. Sequence analysis was performed with the Sequencher 5.1 program (Genes Codes Corporation, Ann Arbor, MI). Two authors masked to each other (J.A.T.

Table 1. Characteristics of the 148 Enrolled Patients with Uveal Melanoma at the Time of Diagnosis

\begin{tabular}{lc}
\hline \multicolumn{1}{c}{ Characteristic } & No. (\%) or Median (Range) \\
\hline No. of patients & $148(100)$ \\
Male & $79(53)$ \\
Female & $69(47)$ \\
Median age, yrs & $63(20-89)$ \\
Median thickness, mm & $4.1(0.3-18.1)$ \\
Median LBD, mm & $11.7(1.5-20.7)$ \\
TNM category & $55(37)$ \\
T1 & $42(28)$ \\
T2 & $40(27)$ \\
T3 & $11(7)$ \\
T4 & \\
TNM stage & $53(36)$ \\
I & $35(24)$ \\
IIA & $29(20)$ \\
IIB & $18(12)$ \\
IIIA & $4(3)$ \\
IIIB & $1(1)$ \\
IIIC & $3(2)$ \\
IV & $5(3)$ \\
Not defined* & \\
LBD = largest basal diameter; TNM = tumor-node- metastasis. \\
*TNM stage is not defined for iris melanoma. \\
\hline
\end{tabular}

and S.M.) independently read sequences. All found mutations were compared with the human reference sequence (GRCh37) and variation database using the University of California Santa Cruz Genome Browser (available at: genome.ucsc.edu). Variant allele frequencies were determined using the Exome Aggregation Consortium (ExAC) database version 0.2 (available at: exac.broadinstitute.org) containing whole-exome variant information for 61748 individuals worldwide, including more than 3300 Finnish individuals from the Sequencing Initiative Suomi project (http:// www.sisuproject.fi). In silico predictions regarding protein function were performed with PolyPhen2, ${ }^{16}$ MutationAssessor, ${ }^{17}$ SIFT, ${ }^{18}$ PROVEAN, ${ }^{19}$ and MutationTaster, ${ }^{20}$ and regarding splice alterations with NNSPLICE0.9 ${ }^{21}$ and NetGene2. ${ }^{22}$ The American College of Medical Genetics and Genomics guidelines were applied to the interpretation of sequence variants. ${ }^{2 .}$

\section{Results}

The median age at diagnosis of the 148 patients was 62 years, and $28(19 \%)$ of them were 50 years of age or younger; the median age of 40 patients who could not be enrolled was 68 years. None had bilateral uveal melanoma. The location of the tumor was the choroid or ciliary body in 143 eyes and the iris in 5 eyes. The tumor-node-metastasis stage distribution was essentially similar to that previously published. ${ }^{24,25}$ The median tumor thickness and largest basal diameter were 6.2 and $12.9 \mathrm{~mm}$, respectively.

A pathogenic variant in $B A P l$ was identified in 3 patients (Fig 1), giving a frequency of $2.0 \%(3 / 148 ; 95 \%$ confidence interval [CI], 0.4-5.8) in this population-based Finnish cohort of patients with uveal melanoma. Calculated for patients 50 years of age or younger or those with bilateral uveal melanoma, the $B A P 1$ pathogenic variant frequency was 1 of 28 patients $(3.6 \%$; $95 \%$ CI, $0.1-18)$. Of the 9 patients from 8 families with a positive family history of uveal melanoma, 2 patients from 2 families carried a $B A P 1$ germline pathogenic variant. The pathogenic variant frequency among the 135 patients with sporadic uveal melanoma was $0.7 \%(95 \% \mathrm{CI}$, 0.0-4.1). Of 141 patients with no BAPl germline mutation, so far 17 have demonstrated metastatic uveal melanoma. Their median age was 66 years, the median tumor thickness was $9.5 \mathrm{~mm}$, and the median largest basal tumor diameter was $14.0 \mathrm{~mm}$.

Two patients (Table 2) carried an identical single nucleotide insertion (T) in exon 14 (c.1780_1781insT, p. G594Vfs*49). The insertion leads to a frame shift and a premature stop codon after 49 amino acids. These 2 patients were not known to be related, but they come from the same geographic region of Finland. The first proband (UMG10-66) had a son (UMG08-58) diagnosed in 2008 at the age of 26 years with a T1a choroidal melanoma that later metastasized (Fig 2, family FUMH-03). The son was tested and also found to carry the c.1780_1781insT mutation. His brother was diagnosed with sarcoma at the age of 63 years and died without being tested. Another brother was diagnosed with renal cancer at the age of 60 years and with prostate cancer at 68 years. The mother of the proband had breast cancer diagnosed at the age of 68 years, and the grandmother had a history of uveal melanoma at the age of 67 years with later metastases; her chart had been discarded, however, so details were not available. The family of the second proband (UMG11-51) also revealed other cancers (Fig 2, family FUMH-08). His deceased father had mesothelioma at the age 61 years, and his grandfather had a T2a choroidal melanoma enucleated at the age of 56 years that later also metastasized. The c.1780_1781insT mutation was not present in the ExAC database. The mutation has not been described previously.

The third patient (UMG12-01; Table 2) identified with a BAPI pathogenic variant had a heterozygous G-to-T transversion in the canonical splice donor in intron $2(\mathrm{c} .67+1 \mathrm{G} \rightarrow \mathrm{T})$, potentially 


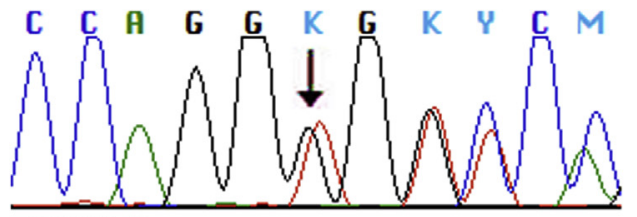

UMG 08-58
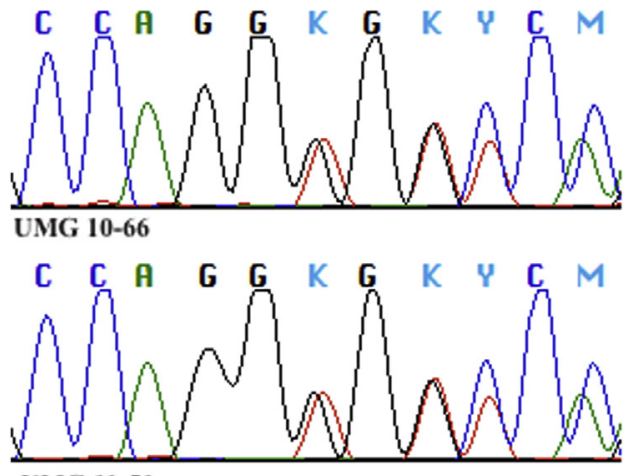

UMG 11-51

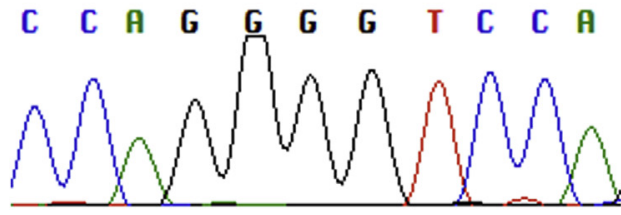

Control

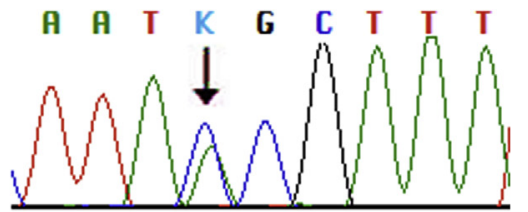

UMG 12-01

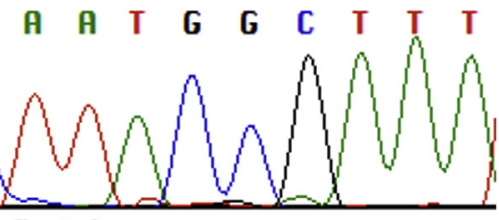

Control

Figure 1. Sequence chromatograms of the 2 BAP1 pathogenic variants. The arrows show the mutations. A, Frameshift mutation in 2 patients (UMG10-66 and UMG11-51) and a control sequence. UMG08-58 is the son of UMG10-66, also with the pathogenic variant. B, Splice donor site mutation $(\mathrm{UMG} 12-01)$ in intron 2 (c.67+1G $\rightarrow \mathrm{T})$ and control sequence. leading to incorrect splicing of BAPl mRNA and, consequently, a nonfunctional protein. Using prediction software, the mutation was predicted to result in loss of the splice donor site with no new donor splice site predicted nearby. The patient also had been diagnosed with bladder cancer at the age of 55 years, and has now demonstrated metastatic uveal melanoma. His family history showed other cancers (Fig 2, family BUMH-01). One deceased brother was diagnosed with bladder cancer and lung cancer, both at the age of 61 years. Another deceased brother was diagnosed with mesothelioma at the age of 47 years. His daughter was diagnosed with renal cancer at 45 years of age and with a neuroendocrine carcinoma of the appendix at 61 years of age. One sister of the proband had breast cancer and the mother had cutaneous melanoma. The $\mathrm{c} .67+1 \mathrm{G} \rightarrow \mathrm{T}$ variant was not present in the ExAC database. The variant has not been described previously.

In addition to the 2 pathogenic variants, we found a nonsynonymous coding variant (c.1689C $\rightarrow$ A; p.His563Gln) of uncertain significance in 1 65-year-old patient with sporadic uveal melanoma. Four programs (PolyPhen, SIFT, PROVEAN, and MutationAssessor) predicted in silico that the variant is nondeleterious to the protein function, although MutationTaster software predicted it to be possibly disease causing. The patient's family history did not reveal any malignancies in close relatives: his father, mother, and only brother did not have any cancers. The variant was not present in the dbSNP (http://www.ncbi.nlm.nih.gov/SNP/), but 1
Finnish carrier was found in the ExAC database (allele frequency, 0.00015).

Further, one insertion carrier (UMG10-66) had a nonsynonymous coding variant $(\mathrm{c} .1727 \mathrm{C} \rightarrow \mathrm{T}$; p.Thr576Ile) of uncertain significance. This variant was not found in his mother, who carried the insertion mutation, and it was predicted in silico to be nondeleterious to the protein function with the same 4 programs as the other variant, but MutationTaster again flagged it as possibly disease causing. It was found in dbSNP (rs374920141) with an allele frequency of 0.00013 .

\section{Discussion}

Herein we report sequencing analysis of the $B A P l$ gene in 148 Finnish uveal melanoma patients drawn from a population-based, consecutive cohort treated from January 2010 through December 2012. The inclusion and participation rates were high at $79 \%$ and $90 \%$, respectively. Altogether, we found 2 different germline pathogenic variants in BAPl in 3 patients, 1 of whom was 50 years old or younger. Two of them had a family history of uveal melanoma. All 3 patients were younger than the median age of our consecutive series. The overall pathogenic variant

Table 2. Characteristics of the Germline BAP1 Pathogenic Variant Carriers

\begin{tabular}{|c|c|c|c|c|c|c|}
\hline Patient & Gender & Age (yrs) & $\begin{array}{l}\text { Thickness } \\
\text { (mm) }\end{array}$ & $\begin{array}{l}\text { Largest Basal } \\
\text { Diameter }(\mathrm{mm})\end{array}$ & $\begin{array}{c}\text { Tumor-Node-Metastasis } \\
\text { Category }\end{array}$ & $\begin{array}{c}\text { Tumor-Node-Metastasis } \\
\text { Stage }\end{array}$ \\
\hline UMG10-66 & Female & 59 & 4.5 & 12.5 & $\mathrm{~T} 2 \mathrm{~b}$ & IIB \\
\hline UMG11-51 & Male & 34 & 10.6 & 16.5 & $\mathrm{~T} 3 \mathrm{~b}$ & IIIA \\
\hline UMG12-01 & Male & 57 & 3.9 & 14.0 & $\mathrm{~T} 2 \mathrm{a}$ & IIA \\
\hline
\end{tabular}



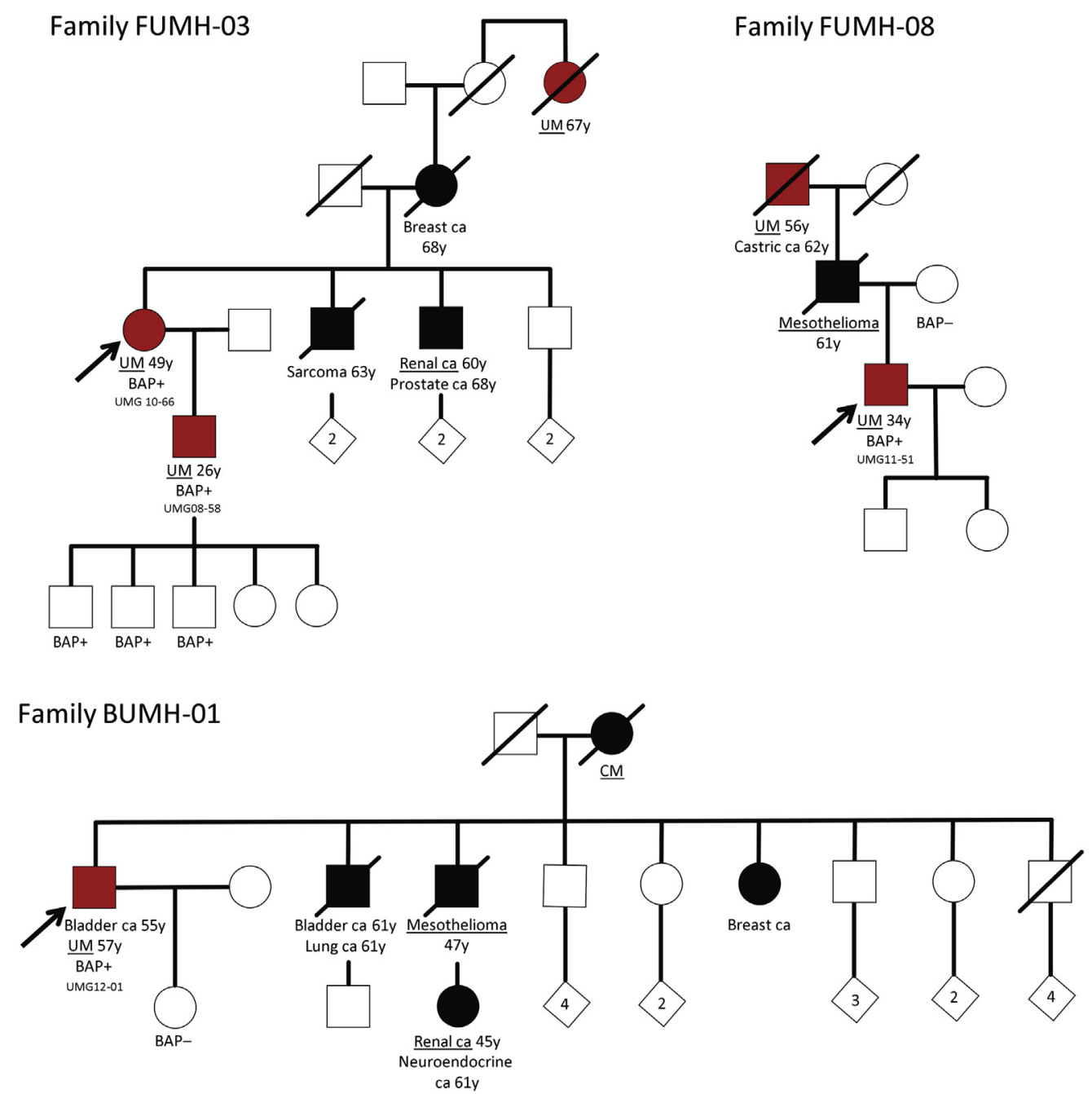

Figure 2. Pedigrees showing the families of BAP1 pathogenic variant carriers. The cancer type, age at diagnosis, and the BAP1 pathogenic variant status when tested are shown. Core components of the BAP1 cancer predisposition syndrome are underlined. UMG\# is an institutional patient identifier. Uveal melanoma patients with or without other cancers are shown in red and patients with other cancers only in black. BAP $+=B A P 1$ pathogenic variant present; $\mathrm{BAP}-=\mathrm{BAP} 1$ pathogenic variant absent; $\mathrm{ca}=$ carcinoma; $\mathrm{CM}=$ cutaneous melanoma; $\mathrm{UM}=$ uveal melanoma; $\mathrm{y}=$ years of age.

frequency of $2.0 \%$ is comparable with that in a referralbased study of North American patients, with a frequency of $1.6 \%$ (95\% CI, 0.7-3.1). ${ }^{14}$ Moreover, the frequency of $3.6 \%$ among patients 50 years of age or younger or with bilateral uveal melanoma was comparable with a population-based Australian study of 66 such patients with a frequency of $3.0 \%{ }^{15}$ (95\% CI, 0.4-10.5). Finally, among 14 selected American patients 30 years of age or younger, a germline BAPl pathogenic variant was found in 1 patient (7\%; 95\% CI, 0.2-34) $)^{26}$ as compared with neither of the 2 patients of this age group in our series; however, a relative of 1 of the $3 B A P 1$ pathogenic variant carriers had been diagnosed with uveal melanoma at 26 years of age. The mutation frequencies consequently were not notably higher in our population that comes from a higherincidence region for uveal melanoma than Northern America, comparable with Australia, where a mean agestandardized incidence of 7 per 1 million has been reported. ${ }^{27}$ In families with 2 or more patients with uveal melanoma, a germline BAPI mutation was reported variably in 4 of 6 Danish families, ${ }^{28}$ as well as in 1 of 5 and in 2 of 11 families in 2 North American series, ${ }^{10,14}$ as compared with 2 of 8 Finnish families in our series.

The strength of our study is its good coverage of Finnish uveal melanoma patients between 2010 and 2012, because all patients are treated centrally in Helsinki University Hospital, a national referral center for this cancer. Furthermore, the participation rate was good. However, our estimate of pathogenic variant frequency may be biased downward because 23 patients had died before they could give consent to this study. Eighteen of these patients had an aggressive form of uveal melanoma with metastasis and could potentially harbor $B A P 1$ germline mutations more often than average. Indeed, in 1 study, mutations were detected in 4 of 50 patients (8\%; 95\% CI, 2-19) in whom metastatic uveal melanoma had developed, as opposed to 
none of 50 matched patients (95\% CI, 0-7) with comparable tumors and follow-up but no metastases. ${ }^{29}$ If we accept an $8 \%$ frequency among patients with metastases, we would have missed up to 2 germline mutations, which would have given an overall pathogenic variant frequency of 5 in 165 patients $(3.0 \%$; 95\% CI, 1.0-6.9). However, of the 3 $B A P 1$ pathogenic variant carriers that we detected, only 1 so far has demonstrated metastases, whereas 18 patients without a germline BAPl mutation by now have been diagnosed with metastatic uveal melanoma.

A second potential limitation is that we sequenced only the coding regions and nearby intronic areas. We could have missed intronic, promoter, and other regulatory area mutations. To date, all published germline BAPI pathogenic variants similarly have been in coding regions or nearby intronic splice sites. No studies so far have sequenced the whole genomic region of the BAPl gene.

The novel insertion mutation in exon 14 (c.1780_1781insT) results in an abnormal protein product with an incorrect and truncated amino acid sequence at the C-terminus. Many important BAP1 protein structures are located downstream from the altered reading frame, including the BRCA1-binding domain, the Yin and Yang 1-binding domain, and nuclear localization signal. ${ }^{30}$ To function properly as a tumor suppressor, BAP1 needs the nuclear localization signal. ${ }^{31}$ Further, BAP1 forms a protein complex with the host cell factor 1 and Yin and Yang 1. The depletion of the Yin and Yang 1-binding domain could interrupt the function of this protein complex as a cell cycle regulator. ${ }^{32}$ Deletion of the BRCA1binding domain abolishes the BAP1-BRCA1 interaction. It is also possible that the quality control system of the cell recognizes the misfolded BAP1 protein and degrades it. ${ }^{33}$

The splice donor site mutation in intron $2(\mathrm{c} .67+1)$ also was novel. The splice donor site includes an almost invariant sequence GU at the $5^{\prime}$ end of the intron. Normally, the spliceosome recognizes the canonical sequence. The mutation likely leads to incorrect splicing of the BAPI mRNA with a possible consequence of deletion of exons. ${ }^{34}$ This presumably also leads to degradation of the mRNA, protein product, or both.

However, we classified 2 nonsynonymous coding variants $\quad(\mathrm{c} .1689 \mathrm{C} \rightarrow \mathrm{A} ; \quad$ p.His563Gln and $\mathrm{c} .1727 \mathrm{C} \rightarrow \mathrm{T}$; p.Thr576Ile) as variants of uncertain significance according to the American College of Medical Genetics and Genomics guidelines. The former was detected in a 65 -year-old patient without any history of cancer in close relatives, and only 1 of 5 prediction programs considered it as possibly disease causing. The latter occurred in a patient carrying a pathogenic variant so that the family history was not informative, but only 1 of 5 prediction programs considered it as possibly disease causing.

A recent review of cancers of $B A P 1$ mutation carriers identified uveal melanoma, mesothelioma, cutaneous melanoma, and renal cell cancer as the 4 most common types. ${ }^{12}$ In support of their role in causing disease, the family histories of the BAPl pathogenic variant carriers revealed at least 2 of these malignancies in each of their families, but we also found additional cancers (e.g., sarcoma and neuroendocrine tumor) that currently are not considered to be core components of the syndrome. In future, we will gain a more precise picture of the BAP1 cancer predisposition syndrome when more patients with pathogenic variants are found and followed up over the long term. In clinical practice, sequencing the $B A P 1$ gene in patients who have a family history of uveal melanoma or other typical BAP1 syndrome-related cancers is rational and also is supported by our data. However, the frequency of germline $B A P 1$ pathogenic variants among patients with sporadic uveal melanoma without family history is 3 times lower.

In conclusion, we show herein that the germline $B A P I$ pathogenic variants are not present in a Finnish populationbased sample of uveal melanoma patients more often than has been reported from other regions. We report 2 novel germline $B A P 1$ pathogenic variants and 3 additional families. Family history of uveal melanoma and of other cancers related to the BAP1 predisposition syndrome should be obtained from all patients with uveal melanoma, and if relevant, $B A P 1$ gene sequencing should be offered. Those who are found to carry a pathogenic variant and their families should be referred for genetic counselling. ${ }^{12}$

Acknowledgments. The authors thank Mikko Muona, MSc, for his assistance with bioinformatics, Sinikka Lindh for laboratory assistance, the participating patients and their families, and Hellevi Saturi for clinical assistance.

\section{References}

1. Kivelä T. The epidemiological challenge of the most frequent eye cancer: retinoblastoma, an issue of birth and death. Br J Ophthalmol 2009;93:1129-31.

2. Kujala E, Mäkitie T, Kivelä T. Very long-term prognosis of patients with malignant uveal melanoma. Invest Ophthalmol Vis Sci 2003;44:4651-9.

3. Kivelä T. Incidence, prevalence and epidemiology of ocular melanoma. In: Murray TG, Boldt HC, eds. Ocular Melanoma: Advances in Diagnostic and Therapeutic Strategies. 1st ed. London: Future Medicine Ltd; 2014:20-38.

4. Virgili G, Gatta G, Ciccolallo L, et al. Incidence of uveal melanoma in Europe. Ophthalmology 2007;114:2309-15.

5. Singh AD, Shields CL, De Potter P, et al. Familial uveal melanoma. Clinical observations on 56 patients. Arch Ophthalmol 1996;114:392-9.

6. Barker-Griffith AE, Streeten BW. Familial uveal melanoma: a report of four cases in two families and literature review. Can J Ophthalmol 2004;39:403-8.

7. Prescher G, Bornfeld N, Hirche H, et al. Prognostic implications of monosomy 3 in uveal melanoma. Lancet 1996;347:1222-5.

8. Damato B, Dopierala JA, Coupland SE. Genotypic profiling of 452 choroidal melanomas with multiplex ligation-dependent probe amplification. Clin Cancer Res 2010;16:6083-92.

9. Harbour JW, Onken MD, Roberson ED, et al. Frequent mutation of BAP1 in metastasizing uveal melanomas. Science 2010;330:1410-3.

10. Abdel-Rahman MH, Pilarski R, Cebulla CM, et al. Germline BAP1 mutation predisposes to uveal melanoma, lung adenocarcinoma, meningioma, and other cancers. J Med Genet 2011;48:856-9.

11. Testa JR, Cheung M, Pei J, et al. Germline BAP1 mutations predispose to malignant mesothelioma. Nat Genet 2011;43: 1022-5. 
12. Rai K, Pilarski R, Cebulla CM, et al. Comprehensive review of BAP1 tumor predisposition syndrome with report of two new cases. Clin Genet 2015 Jun 22. doi: 10.1111/cge.12630.

13. Field MG, Harbour JW. Recent developments in prognostic and predictive testing in uveal melanoma. Curr Opin Ophthalmol 2014;25:234-9.

14. Gupta MP, Lane AM, DeAngelis MM, et al. Clinical characteristics of uveal melanoma in patients with germline BAP1 mutations. JAMA Ophthalmol 2015;133:881-7.

15. Aoude LG, Vajdic CM, Kricker A, et al. Prevalence of germline BAP1 mutation in a population-based sample of uveal melanoma cases. Pigment Cell Melanoma Res 2013;26:278-9.

16. Adzhubei IA, Schmidt S, Peshkin L, et al. A method and server for predicting damaging missense mutations. Nat Methods 2010;7:248-9.

17. Reva B, Antipin Y, Sander C. Predicting the functional impact of protein mutations: application to cancer genomics. Nucleic Acids Res 2011;39:e118.

18. Kumar P, Henikoff S, Ng PC. Predicting the effects of coding non-synonymous variants on protein function using the SIFT algorithm. Nat Protoc 2009;4:1073-81.

19. Choi Y, Sims GE, Murphy S, et al. Predicting the functional effect of amino acid substitutions and indels. PLoS One 2012;7:e46688.

20. Schwarz JM, Cooper DN, Schuelke M, et al. MutationTaster2: mutation prediction for the deep-sequencing age. Nat Methods 2014;11:361-2.

21. Reese MG, Eeckman FH, Kulp D, et al. Improved splice site detection in genie. J Comput Biol 1997;4:311-23.

22. Hebsgaard SM, Korning PG, Tolstrup N, et al. Splice site prediction in Arabidopsis thaliana pre-mRNA by combining local and global sequence information. Nucleic Acids Res 1996;24:3439-52.

23. Richards S, Aziz N, Bale S, et al. Standards and guidelines for the interpretation of sequence variants: a joint consensus recommendation of the American College of Medical Genetics and Genomics and the Association for Molecular Pathology. Genet Med 2015;17:405-24.

24. Kujala E, Damato B, Coupland SE, et al. Staging of ciliary body and choroidal melanomas based on anatomic extent. J Clin Oncol 2013;31:2825-31.

25. AJCC Ophthalmic Oncology Task Force. International validation of the American Joint Committee on Cancer's 7th edition classification of uveal melanoma. JAMA Ophthalmol 2015;133:376-83.

26. Cebulla CM, Binkley EM, Pilarski R, et al. Analysis of BAP1 germline gene mutation in young uveal melanoma patients. Ophthalmic Genet 2015;36:126-31.

27. Vajdic CM, Kricker A, Giblin M, et al. Incidence of ocular melanoma in Australia from 1990 to 1998. Int J Cancer 2003; 105:117-22.

28. Wadt KA, Aoude LG, Krogh L, et al. Molecular characterization of melanoma cases in Denmark suspected of genetic predisposition. PLoS One 2015;10:e0122662.

29. Njauw CN, Kim I, Piris A, et al. Germline BAP1 inactivation is preferentially associated with metastatic ocular melanoma and cutaneous-ocular melanoma families. PLoS One 2012;7: e35295.

30. Carbone M, Yang H, Pass HI, et al. BAP1 and cancer. Nat Rev Cancer 2013;13:153-9.

31. Ventii KH, Devi NS, Friedrich KL, et al. BRCA1-associated protein-1 is a tumor suppressor that requires deubiquitinating activity and nuclear localization. Cancer Res 2008;68:6953-62.

32. Yu H, Mashtalir N, Daou S, et al. The ubiquitin carboxyl hydrolase BAP1 forms a ternary complex with YY1 and HCF1 and is a critical regulator of gene expression. Mol Cell Biol 2010:30:5071-85.

33. Chen B, Retzlaff M, Roos T, et al. Cellular strategies of protein quality control. Cold Spring Harb Perspect Biol 2011;3: a004374.

34. Hoskins AA, Moore MJ. The spliceosome: a flexible, reversible macromolecular machine. Trends Biochem Sci 2012;37:179-88.

\section{Footnotes and Financial Disclosures}

Originally received: September 10, 2015.

Final revision: December 17, 2015.

Accepted: January 6, 2016.

Available online: February 12, 2016.

Manuscript no. 2015-1574

${ }^{1}$ Department of Ophthalmology, University of Helsinki and Helsinki University Hospital, Helsinki, Finland.

${ }^{2}$ Folkhälsan Institute of Genetics, Helsinki, Finland.

${ }^{3}$ Department of Medical Genetics, University of Helsinki and Helsinki University Hospital, Helsinki, Finland.

${ }^{4}$ Neuroscience Center and Research Programs Unit, Molecular Neurology, University of Helsinki, Helsinki, Finland.

Presented at: European Association for Vision and Eye Research Annual Meeting, October 2014, Nice, France; and International Society of Ocular Oncology Annual Meeting, June 2015, Paris, France.

*Both Joni A. Turunen, MD, PhD, and Salla Markkinen, MSc, contributed equally as first authors.

Financial Disclosure(s):

The author(s) have no proprietary or commercial interest in any materials discussed in this article.

Supported by the Mary and Georg C. Ehrnrooth Foundation (Helsinki, Finland); the Sigrid Jusélius Foundation; the Eye and Tissue Bank Foundation (Helsinki, Finland); the Folkhälsan Research Foundation (Helsinki,
Finland); the Finnish Ophthalmological Society (Ylikiiminki, Finland); the Evald and Hilda Nissi Foundation (Espoo, Finland); and the Paulo Foundation (Helsinki, Finland). The funding organizations had no role in the design or conduct of this research.

Author Contributions:

Conception and design: Turunen, Lehesjoki, Kivelä

Analysis and interpretation: Turunen, Markkinen, Saarinen, Lehesjoki, Kivelä

Data collection: Turunen, Markkinen, Wilska, Saarinen, Raivio, Täll, Kivelä

Obtained funding: Turunen, Markkinen, Kivelä

Overall responsibility: Turunen, Markkinen, Wilska, Saarinen, Raivio, Täll, Lehesjoki, Kivelä

Abbreviations and Acronyms:

BAP1 = BRCA1-associated protein-1; BRCA1 = breast cancer 1; CI $=$ confidence interval $; \mathbf{E x A C}=$ Exome Aggregation Consortium; LBD $=$ largest basal diameter; TNM $=$ Tumor, Node, Metastasis classification; UCSC $=$ University of California Santa Cruz

Correspondence:

Joni A. Turunen, MD, PhD, Department of Ophthalmology, University of Helsinki and Helsinki University Hospital, Haartmaninkatu 4C, FI-00029 HUS, Helsinki, Finland. E-mail: joni.turunen@ helsinki.fi. 